\title{
Confiabilidade dos dados do Sistema de Informações sobre Nascidos Vivos em Teresina, Estado do Piauí, Brasil - 2002
}

\author{
Reliability of data available in the Information System \\ for Live Birth in the city of Teresina, Piauí State, Brazil - 2002
}

M árcio Dênis M edeiros M ascarenhas ${ }^{1}$

Keila Rejane Oliveira Gomes ${ }^{1}$

${ }^{1}$ UniversidadeFederal do

Piauí. Campus M inistro

Petrônio Portela, Iningá

64049-550 Teresina PI.

mdm.mascarenhas@gmail.com
Abstract This article aims to evaluate the reliability of the data available in the Information System for Live Birth (SINASC) in the city of Teresina, Piauí State, Brazil for the year of 2002. The proportion of data completeness was analyzed, and concordance between variables for data reported to SINASC and hospital records (SINASC-P) was estimated using the kappa index. This study demonstrated that variables in SINASC presented a higher level of completeness ( $>90 \%$ ) than those in SINASC-P (>74\%), and the concordance was excellent (kappa>0.80) for the following variables: maternal age, marital status, number of live births, type of delivery, and the sex and weight of newborn infants. Concordance for the number of stillbirths, prenatal visits, educational level and gestational age was categorized from fair-to-adequate $(0.20<$ kappa<0.80). SI NASC variablesshowed excellent completenessand good (kappa=0.76) general reliability. Werecommend improvement in the quality of data collection in hospital records and SINASC in Teresina. Key words Live birth, Birth certificates, Health information systems
Resumo Este artigo tem como objetivo avaliar a confiabilidade dos dados do Sistema de Informações sobre N ascidos Vivos (SINASC) em Teresina, Piauí, no ano de 2002. Estudou-se a proporção de preenchimento e a concordância entre as variáveis do SINASC e dos registros hospitalares (SIN ASC-P), utilizando-se o índice kappa. As variáveis do SINASC apresentaram melhor qualidade de preenchimento (completude $>90 \%$ ) em relação às variáveis do SINASC-P (completude>74\%). A concordância foi excelente (kappa $>0,80$ ) para as variáveis idade da mãe, estado civil, número de filhos nascidos vivos, tipo de parto, sexo e peso do recém-nascido. As variáveis número de filhos nascidos mortos, consultas prénatal, escolaridade e duração da gestação apresentaram concordância, variando entremínima eboa $(0,20<k a p p a<0,80)$. O SIN ASC apresentou excelentequalidadede preenchimento e concordância média considerada boa (kappa=0,76). Recomenda-se melhorar a qualidade dos registros hospitalares eaprimorar a coleta de dados do SINASC em Teresina.

Palavras-chave N ascido vivo, D eclaração denascimento, Sistemas de informação em saúde 


\section{Introdução}

Obter informações que subsidiem a tomada de decisões para melhorar o nível de saúde de uma população é de fundamental importância para gestores de saúde, pois esse conhecimento aplica-se ao planejamento, à organização e avaliação de ações eserviços de saúde. Para fundamentar 0 diagnóstico de situação de saúde, é necessário que os tomadores de decisão apropriem-se de informações confiáveis sobre aspectos como 0 perfil da morbidadee mortalidade, fatores derisco eseus determinantes, características demográficas e serviços de assistência médico-sanitária ${ }^{1,2}$. Dispor de boas estatísticas sobre os eventos vitais contribui para o desenvolvimento de melhorias na saúde da população e divulgá-las favorece o controle social das ações implantadas. Todavia, a informação em saúde nem sempre apresenta boa qualidade, a qual depende diretamente da cobertura dos eventos registrados e da fidedignidade dos dados coletados $5^{3,4}$.

No Brasil, tem-se assistido a uma crescente geração de dados e constituição de sistemas de informações com os mais diversos fins, principalmente a partir das últimas décadas do século $X X$. Os sistemas de informação em saúde (SIS) foram implantados na década de noventa em todas as capitais de estado, com a finalidade de gerar dados epidemiológicos que pudessem subsidiar a tomada de decisão por aqueles que planejam e executam as políticas e ações de saúde no país. $\mathrm{Na}$ área materno-infantil, implantou-se 0 Sistema de Informações sobre $\mathrm{N}$ ascidos Vivos (SIN ASC) com o objetivo de obter dados sobreo número de nascimentos, condições do parto e 0 perfil epidemiológico demãeserecém-nascidosa partir da Declaração de Nascido Vivo (DN) 5,6.

Os dados do SIN ASC têm sido objeto de estudo não somente para obter o perfil da população materno-infantil, como apontam os resultados das primeiras pesquisas contemporâneas à sua implantação $0^{5-9}$, mas também para avaliar aspectos como cobertura dos nascimentos equalidade da informação gerada. Citam-se diversos estudos nacionais ${ }^{10-16}$ que adotaram distintas metodologias para dimensionar tais aspectos como, por exemplo, avaliação da completude dos dados, verificação da confiabilidade dos registros por meio de comparação com outras fontes de dados (prontuários hospitalares, entrevistas individualizadas) ou validação de dados para obter medidas de sensibilidade e especificidade a partir de estudos do tipo caso-controle.
Sabe-se que a falta de informações confiáveis pode comprometer tanto a formulação de indicadores desaúdeeestudos epidemiológicos quanto a implementação de medidas para mel horar 0 nível de vida da população. Embora exista uma avaliação sobre a qualidade da informação do SINASC quanto à clareza metodológica da documentação, completude do preenchimento e consistência para o país, grandes regiões e unidades da federação no ano de $2002^{13}$, ainda são escassas as avaliações no âmbito local.

Considerando a ausência de análises sobre a qualidade de dados do SINASC do município de Teresina (PI), é que se propõe o presente artigo. Dada à originalidade da pesquisa para o município e para o estado, seus resultados poderão ser úteis aos gestores das instituições que geram dados para o SINASC a fim de que, uma vez identificadas as inconsistências, busquem suas causas e invistam em soluções que resultem em maior confiabilidade da informação produzida sobre nascidos vivos.

\section{Métodos}

0 presenteestudo constitui-seem um subprojeto da "Avaliação da Qualidade dos Dados do Sistema de Informações sobre Nascidos Vivos em Te resina, Piauí", cujos detalhes metodológicos e re sultados iniciais foram apresentados por Gomes et al. ${ }^{17}$. Trata-se de um estudo descritivo, de abordagem quantitativa, realizado no município de Teresina (população residente em 2002: 740.016 habitantes) ${ }^{18}$, capital do Estado do Piauí. O btevese o banco de dados oficial do SINASC de quatro maternidades públicas selecionadas $(A, B, C$ eD), nas quais ocorreram $83 \%$ dos 14.413 nascidos vivos (NV), filhos demães residentes em Teresina no período de janeiro a dezembro de 2002. Devido ao elevado número de partos e à ausência de variação apreciável na ocorrência denascimentos duranteo ano, realizou-sesorteio do tipo simples para identificar, em cada maternidade selecionada, um único mês (exceto fevereiro) de ocorrência dos nascimentos, cujos dados seriam coletados $^{19}$. Não foram observadas modificações nas rotinas dos serviços incluídos no estudo, como greves ou mudança na composição das equipes médicas ou de enfermagem, que pudessem interferir nos resultados do estudo.

A partir de consulta a prontuários e livros de registros, criou-se o Sistema de Informações sobre Nascidos Vivos Pesquisa (SINASC-P), cujos 
dados foram coletados por meio do formulário DN -Pesquisa, elaborado com base no modelo da DN oficial, obedecendo à mesma sistemática $e$ categorização do SINASC. A principal fonte de dados foi o prontuário. Quando este não era localizado ou quando não apresentava todos os dados necessários à pesquisa, consultaram-se os livros de registro da sala de parto ou do centro cirúrgico.

Nos meses sorteados para cada maternidade, foi registrada a soma de $970 \mathrm{NV}$ filhos de mães residentes em Teresina e que apresentavam tipo de gravidez única. As informações foram coletadas a partir de consulta exclusiva a prontuários para 834 (86,2\%) NV, com base apenas nos livros de registro para 107 (11,1\%) NV epor meio de prontuários e complementadas com dados dos livros de registro para $26(2,7 \%)$ outros. Assim, 967 (99,7\%) NV tiveram suas informações coletadas, ao passo que três NV não foram localizados em nenhuma das fontes de dados, resultando em perda de $0,3 \%$.

$\mathrm{N}$ a rotina dos serviços incluídos no estudo, 0 preenchimento da DN, prontuários e livros de registros era realizado tanto pela equipe médica quanto pelos profissionais de enfermagem. A informação sobre a idade gestacional (IG) do recém-nascido era registrada pelos neonatologistas com base no método de Capurro et al. ${ }^{20}$. Quando o registro da IG não constava do prontuário, anotavam-se as informações dos laudos de ultrassonografia para realizar o cálculo da IG, à data do nascimento do recém-nascido, conforme metodologia apresentada por Gomes et al. ${ }^{17}$. Quando não encontrada qualquer informação sobre a IG estimada pelo método de Capurro ou pela ultrassonografia, a IG era calculada a partir da data da última menstruação (DUM) informada nos registros hospitalares. Segundo critério utilizado por Silva et al. ${ }^{10}$, quando não se obteve o dia, mas apenas o mês em que ocorreu a última menstruação, foi imputado o dia 15. Finalmente, quando não foi possível obter nem dia nem mês da última menstruação, a IG foi considerada ignorada.

Dada à metodologia adotada e por meio da aplicação do pré-teste, decidiu-se que seriam consideradas como verdadeiras as informações contidas nos registros hospitalares, a exemplo de outros estudos disponíveisnaliteratura específica ${ }^{3,10,11}$.

Os dados do SINASC-P foram digitados no programa Epi Info 6.04. Uma vez selecionados os registros de nascimentos ocorridos no mês sorteado para cada maternidade, o banco de da- dos do SINASC foi exportado para o mesmo programa, obtendo-se, desta forma, dois bancos de dados com igual número de registros. $A$ análise do preenchimento das variáveis baseouse na classificação sugerida por M ello Jorge et al. 7 : preenchimento excelente (percentual debranco/ignorado $<10 \%$ ), bom (percentual de branco/ignorado entre $10 \%$ e $29,9 \%$ ) ou ruim (percentual de branco/ignorado $\geq 30 \%$ ). Para a análise de concordância entreos dados, utilizou-seo índice kappa, que compara a diferença entre as concordâncias observada e esperada, chegando ao valor máximo possível para esta diferença, podendo variar entre-1 (discordância perfeita) e 1 (concordância perfeita) ${ }^{21}$. Os valores de kappa, calculados no programa SPSS for WindoWS, foram interpretados por meio dos seguintes critérios ${ }^{22}$ : concordância desprezível $(<0,20)$; concordância mínima $(0,20$ a 0,40); concordância ruim $(0,41$ e 0,60$)$; concordânciaboa $(0,61$ e0,80); concordância excelente $(0,81$ a 1,00$)$. As variáveis contínuas como número de filhos tidos em gestações anteriores (nascidos vi vos enascidos mortos), idade da mãe e peso ao nascer foram convertidas em variáveis categóricas.

0 projeto de pesquisa foi apresentado aos Comitês de Ética em Pesquisa da Universidade Federal do Piauí e das maternidades envolvidas. Solicitou-se consentimento dos gestores das respectivas maternidades, garantindo-Ihes 0 anonimato das instituições e das pessoas cujos registros compõem os bancos de dados analisados.

\section{Resultados}

As variáveis encontravam-se preenchidas com maior frequência no SINASC, cuja qualidade do preenchimento foi considerada excelente (percentual de branco/ignorado $<10 \%$ ) para as variáveis idade, estado civil, escolaridade, número defilhos nascidos vivos, duração da gestação, tipo de parto, número de consultas de pré natal, sexo do recém-nascido epeso ao nascer. A variável núme ro de filhos nascidos mortos apresentou preenchimento de qualidade boa (percentual de branco/ignorado entre $10 \%$ e 29,9\%). Entre as variáveis do SINASC-P, observou-se o inverso: a maioria delas foi classificada apenas como boa, exceto as variáveis idade da mãe, tipo de parto, sexo do neonato e peso nascer, que apresentaram qualidade de preenchimento excelente (Tabela 1).

No SINASC, dentreas características da mãe, a variável idadeapresentou melhor preenchimen- 
to. Sobre as características da gestação e do parto, a variável melhor preenchida foi duração da gestação. Quanto às características do neonato, a variável peso ao nascer foi a melhor preenchida. Estas variáveis apresentaram $100 \%$ de preenchimento. No SINASC-P, o preenchimento foi excel ente para as variáveis idade da mãe, tipo de parto, sexo do recém-nascido e peso ao nascer. As demais variáveis apresentaram preenchimento avaliado como bom (Tabela 1).

A concordância dos dados foi classificada como excelente (kappa $>0,80)$ para as variáveis idade, estado civil, número de filhos nascidos vivos, tipo de parto, sexo do recém-nascido e peso ao nascer. A variável número de filhos nascidos mortos teve concordância boa $(0,60<k a-$ ppa $<0,80)$. As variáveis escolaridade e número de consultas de pré-natal apresentaram concordância ruim $(0,40<k a p p a<0,60)$. Concordância mínima $(0,20<k a p p a<0,40)$ foi verificada para a variável duração da gestação (Tabela 2).

\section{Discussão}

Embora o SINASC apresente boas taxas de cobertura em relação aos demais sistemas nacionais de informação em saúde, tem-se observado intensificação no desenvolvimento de pesquisas com vistas a avaliar a confiabilidade e validação dos dados sobre nascidos vivos e a identificar a melhor forma de coletá-los, $11,23,24$.

$\mathrm{Na}$ análise dos dados do SIN ASC, a variável número de filhos nascidos mortos apresentou 0 pior preenchimento dentre as demais variáveis estudadas. Em estudo realizado com dados referentes à cidade de Teresina em 1992, esta mesma variável era preenchida em apenas $37 \%$ das DN 7 . A essa época, o SINASC encontrava-se em processo de implantação nos demais municípios do Piauí. Ao comparar os dados obtidos dez anos após este processo, os resultados apontam para a expressiva mel horia no preenchimento das informações, a exemplo do que se verifica em outras regiões do estado e do país ${ }^{11,25}$.

Chama a atenção o percentual de preenchimento das variáveis do SINASC-P, cujos dados foram coletados a partir de consulta aos prontuários elivros de registro. Isto é preocupante, pois se espera que a informação obtida no registro hospitalar seja a mais completa possível. A DN deve ser preenchida com base nos registros hospitalares, principalmente, por meio de consulta ao prontuário e ao cartão da gestante, uma vez que o SINASC foi concebido tendo nos registros hospitalares a principal fonte de obtenção de dados sobre nascidos vivos ${ }^{5}$. No entanto, observa-se que, na rotina das maternidades incluídas
Tabela 1. Frequência e percentual de registros com preenchimento em branco ou ignorado segundo variáveis do SINASC e do SINASC-P. Maternidades selecionadas do município de Teresina (PI), 2002.

\begin{tabular}{|c|c|c|c|c|}
\hline \multirow[b]{2}{*}{ Variáveis } & \multicolumn{2}{|c|}{ SINASC } & \multicolumn{2}{|c|}{ SINASC-P } \\
\hline & $\mathrm{n}$ & $\%$ & $\mathrm{n}$ & $\%$ \\
\hline \multicolumn{5}{|l|}{ Referentes à mãe } \\
\hline Idade & 0 & 0,0 & 0 & 0,0 \\
\hline Estado civil & 1 & 0,1 & 149 & 14,5 \\
\hline Escolaridade & 5 & 0,5 & 158 & 16,3 \\
\hline Filhos nascidos vivos & 65 & 6,7 & 189 & 19,5 \\
\hline Filhos nascidos mortos & 130 & 13,4 & 148 & 15,3 \\
\hline \multicolumn{5}{|c|}{ Referentes à gestação e ao parto } \\
\hline Duração da gestação & 0 & 0,0 & 139 & 14,4 \\
\hline Tipo de parto & 1 & 0,1 & 0 & 0,0 \\
\hline Consultas de pré-natal & 4 & 0,4 & 251 & 26,0 \\
\hline \multicolumn{5}{|l|}{ Referentes ao recém-nascido } \\
\hline Sexo & 6 & 0,6 & 1 & 0,1 \\
\hline Peso ao nascer & 0 & 0,0 & 0 & 0,0 \\
\hline
\end{tabular}

SINASC: Sistema de Informações sobre N ascidos Vivos ( $n=967$ ); SINASC-P: Sistema de Informações sobre N ascidos Vivos-Pesquisa $(n=967)$.
Tabela 2. Concordância entre os dados de variáveis do SINASC e do SINASC-P. Maternidades selecionadas do Município de Teresina (PI), 2002.

\begin{tabular}{lcc}
\hline \multicolumn{1}{c}{ Variáveis } & $\mathrm{n}^{*} \begin{array}{c}\text { Índice kappa } \\
\text { bruto }\end{array}$ \\
\hline $\begin{array}{l}\text { Referentes à mãe } \\
\quad \text { Idade }\end{array}$ & 967 & 0,97 \\
$\quad$ Estado civil & 814 & 0,91 \\
$\quad$ Escolaridade & 804 & 0,42 \\
$\quad$ Filhos nascidos vivos & 726 & 0,87 \\
$\quad$ Filhos nascidos mortos & 705 & 0,62 \\
Referentes à gestação e ao parto & & \\
$\quad$ Duração da gestação & 826 & 0,35 \\
$\quad$ Tipo de parto & 966 & 0,97 \\
$\quad$ Consultas de pré-natal & 715 & 0,51 \\
Referentes ao recém-nascido & & \\
$\quad$ Sexo & 960 & 0,98 \\
Peso ao nascer & 967 & 0,97 \\
\hline
\end{tabular}

SINASC: Sistema de Informações sobre N ascidos Vivos ( $n=967)$; SI N ASC-P: Sistema de Informações sobre N ascidos Vivos-Pesquisa ( $n=967)$.

* Para o cálculo do índice kappa, os casos com informações ignoradas ou sem preenchimento foram excluídos. 
no estudo, a mãe é solicitada a prestar informações durante $o$ trabalho de parto ou imediatamente após a expulsão do recém-nascido. Desta forma, as informações são obtidas erroneamente e em momento inoportuno. Outras vezes, são fornecidas pelo companheiro ou por familiares da puérpera, acarretando ausência de informação ou falha na qualidade da mesma, principalmenteem relação à idadegestacional, quando da ausência de profissionais capacitados ou de recursos específicos ${ }^{26}$.

Embora a informação sobre o número de filhos tidos (vivos e mortos) seja de grande importância para caracterizar a população-alvo do SINASC e para o acompanhamento da taxa de fecundidade, parecehaver pouca conscientização ou pouco treinamento sobre a importância e 0 correto preenchimento deste dado. Quanto à variável número de consultas de pré natal, a ausência desta informação nos registros hospitalares impede o conhecimento sobre grupos populacionais mais vulneráveis ${ }^{11}$.

A variável escolaridade é de difícil obtenção durante a hospitalização, pois, geralmente, não consta dos registros hospitalares. Na DN , ela está categorizada em intervalos de anos de estudo concluídos, enquanto nos prontuários écomum ser categorizada em primeiro grau ou primário, segundo grau ou secundário e terceiro grau ou superior. Tal categorização dificulta ainda mais a obtenção do dado, poisobriga o responsável pelo preenchimento a calcular o número de anos de estudo e escolher a casela correta no modelo vigente da $\mathrm{DN}{ }^{11}$.

O fornecimento de dados para variáveis como escolaridade, duração da gestação e número de consultas de pré-natal requer mais tempo e trabalho por parte dequem preenchea D N. 0 dado pode ter sido anotado nas fontes de registro hospitalar, mas a comodidade de questionar a mãe (muitas vezes no momento do parto) ou familiares faz com que essas variáveis sejam respondidas sob a influência do viés da memória, o que evidencia a fragilidade da informação obtida a partir da análise simples dos dados do SINASC. Alguns estudos alertam para o cuidado com o uso dos registros da DN na identificação de fatores de risco para o óbito infantil devido a falhas no processo de coleta de dados $23,27,28$.

A análise da concordância dos dados foi realizada para as variáveis que se encontravam preenchidas em ambos os bancos de dados. M erece destaque a baixa concordância da variável escolaridade, o que podeser explicado pela dificuldade em obter esta informação a partir da conver- são do nível de ensino em anos de estudo completos. A variável número de filhos nascidos mortos, que apresentou concordância boa, demonstrou-se menos confiável do que a variável número de filhos nascidos vivos, com concordância excelente. Durante a coleta de dados, observou-se que, nos registros hospitalares, a informação sobre fil hos natimortos foi menos valorizada. Em muitas ocasiões, quando existia referência à natimortalidade, a quantidade de fiIhos mortos não era especificada, acarretando dúvida se o não preenchimento indicava ausência defilhos nascidos mortos ou omissão do preenchimento da variável.

0 índice de concordância da variável tipo de parto foi igual à encontrada no município do Rio de Janeiro $(0,97)$ esuperior à de São Luís, no $M$ aranhão $(0,90)^{10,11}$. De maneira inversa, as informações sobre número de consultas de prénatal e duração da gestação mostraram-se me nos confiáveis no município de Teresina em relação aos resultados obtidos naquelas cidades. A variável duração da gestação apresentou o pior índice de concordância, resultado superior ao obtido em São Luís $(0,05)$ ebastantediferente da análise realizada com os registros de nascidos vivos do Rio de Janeiro $(0,73)^{10,11}$. As variáveis referentes ao recém-nascido apresentaram excelente concordância, com valores semelhantes aos resultados obtidos em outros estudos ${ }^{10,11}$. Tal concordância permite a utilização das informações do SIN ASC para as estimativas de baixo peso ao nascer. Ao contrário da idade gestacional, a informação sobre o peso ao nascer consiste em dado acessível e consistente, reafirmando-se como critério para identificação de risco para a mortalidade infantil, quando do planejamento das ações de saúde 6 .

N este estudo, os registros hospitalares encontravam-se mal preenchidos, ou seja, a maior parte das variáveis analisadas, com exceção das variáveis idade da mãe, tipo de parto, sexo do recémnascido e peso ao nascer, apresentou percentual de preenchimento inferior a $90 \%$. Verifica-se, assim, um paradoxo: maior percentual de preenchimento no SINASC, cujos dados originam-se da DN, que deve ser preenchida com base nos prontuários, os quais, por sua vez, apresentaram menor percentual de preenchimento. Logo, neste cenário, não foi possível identificar qual a melhor fonte de dados, pois se podeter um SINASC com informação de qualidade superestimada e registros hospitalares aceitáveis ou o contrário.

Os resultados apontam para a necessária cautela quanto ao uso das informações oficiais so- 
bre nascidos vivos no sentido de que possíveis falhas possam interferir no adequado uso das informações sobre a população materno-infantil, cálculo de taxas e planejamento de ações de atenção à saúde desta parcela populacional. Desperta a atenção dos gestores com relação à manutenção da qualidade dos registros hospitalares e quanto ao aprimoramento das etapas de coleta e processamento dos dados e divulgação das informações em saúde.

É preciso intensificar a orientação das pessoas diretamente envolvidas na coleta de dados, tentando despertar o interesse pela produção de informações fidedignas para a construção de sistemas de informações mais confiáveis, fazendo com que as decisões a serem tomadas sejam subsidiadas por dados que representem a realidade do evento registrado.

\section{Colaboradores}

M DM M ascarenhas participou da concepção teórica, levantamento bibliográfico, consolidação e análise dos dados, redação e revisão final do texto; KRO Gomes contribuiu na concepção teórica, análise dos dados e revisão da versão final.

\section{Agradecimentos}

Aos alunos do curso de Enfermagem da Universidade Federal do Piauí, Elda de Sousa Santos, Grazyella da Paz Santos, Ketiana M elo Guimarães eT iago de Sousa M acêdo, pelo valioso auxílio durante a coleta de dados; à Fundação M unicipal de Saúde de Teresina, pela concessão do banco de dados do SINASC; a Tatiana Miranda Lanzieri e D ouglas H atch, pelo apoio e sugestões durante a redação final do artigo. 
Referências

1. Andrade SM, Soares DA. Dados e informações em saúde: para que servem? In: Andrade SM, Soares DA, Cordoni Jr L, organizadores. Bases da saúde coletiva. Londrina: UEL; 2001. p. 161-182.

2. Mota $E$, Carvalho DMT. Sistemas de informação em saúde. In: Rouquayrol MZ, Almeida Filho N, organizadores. Epidemiologia \& Saúde. 6ª ed. Rio de Janeiro: M edsi; 2003. p. 605-628.

3. Godlee F, Pakenham-Walsh N, N cayiyana D, Cohen $B$, Packer $A$. Can we achieve health information for all by 2015? Lancet 2004; 364:295-300.

4. Szwarcwald CL, Leal MC, Andrade CLT, Souza Jr PRB. Estimação da mortalidade infantil no Brasil: 0 que dizem as informações sobre óbitos e nascimentos do M inistério da Saúde? Cad Saude Publica 2002; 18:1725-1736.

5. Mello Jorge MHP, Gotlieb SLD, Soboll MLMS, AImeida M F, Latorre MRDO. Avaliação do Sistema de Informação sobre $\mathrm{N}$ ascidos Vivos e o uso de seus dados em epidemiologia e estatísticas de saúde. Rev. Saude Publica 1993; 27(Supl.):1-46.

6. Nascimento LFC, Gotlieb SLD. Fatores de risco para o baixo peso ao nascer, com base em informações da Declaração de Nascido Vivo em Guaratinguetá, SP, no ano de 1998. Inf Epidemiol SUS 2001; 10:113120.

7. Mello Jorge MHP, Gotlieb SLD, Oliveira H. O Sistema de Informações sobre Nascidos Vivos: primeira avaliação dos dados brasileiros. Inf Epidemiol SUS 1996; 5:15-48.

8. Rodrigues $C S, M$ agalhães Júnior $H M$, Evangelista PA, Ladeira RM, Laudares S. Perfil dos nascidos vivos no Município de Belo Horizonte, 1992-1994. Cad Saude Publica 1997; 13:53-57.

9. Mello Jorge MHP, Gotlieb SLD, Andrade SM. Análise dos registros de nascimentos vivos em localidade urbana no Sul do Brasil. Rev. Saude Publica 1997; 31:78-89.

10. Silva AAM, Ribeiro VS, Borba Jr AF, Coimbra LC, Silva RA. Avaliação da qualidade dos dados do Sistema de Informações sobre Nascidos Vivos em 1997. 1998. Rev. Saude Publica 2001; 35:508-514.

11. Theme Filha MM, Gama SGN, Cunha CB, Leal MC. Confiabilidade do Sistema de Informações sobre Nascidos Vivos hospitalares no município do Rio de Janeiro, 1999-2001. Cad Saude Publica 2004; 20(Supl.):S83-S91.

12. Almeida M F, Alencar GP, França Júnior I, Novaes $H M D$, Siqueira AAF, Schoeps D, Campbell O, Rodrigues LC. Validade das informações das declarações de nascidos vivos com base em estudo de caso-controle. Cad Saude Publica 2006; 22:643-652.

13. Romero DE, Cunha CB. Avaliação da qualidade das variáveis epidemiológicas e demográficas do Sistema de Informações sobre N ascidos Vivos, 2002. Cad Saude Publica 2007; 23:701-714.

14. M ello Jorge MHP, Laurenti R, Gotlieb SLD. Análise da qualidade das estatísticas vitais brasileiras: a experiência de implantação do SIM e do SINASC. Cien Saude Colet 2007; 12:643-654.
15. Guerra FAR, Llerena Júnior JC, Gama SGN, Cunha $C B$, Theme Filha M M. Confiabilidade das informações das declarações de nascido vivo com registro de defeitos congênitos no Município do Rio de Janeiro, Brasil, 2004. Cad Saude Publica 2008; 24:438-446.

16. Drumond EF, Machado CJ, França E. Subnotificação de nascidos vivos: procedimentos de mensuração a partir do Sistema de Informação Hospitalar. Rev. Saude Publica 2008; 42:55-63.

17. Gomes KRO, Santos ES, Santos GP, M ascarenhas MDM. Qualidade do dado referente à idade gestacional no Sistema de Informação sobre $\mathrm{N}$ ascidos Vivos (SINASC) em Teresina-PI. Rev Baiana Saúde Pública 2006; 30:238-247.

18. Instituto Brasileiro de Geografia e Estatística. População. [site da Internet] [acessado 2007 jul 16]. Disponível em: http://www.ibge.gov.br

19. Silva NN. Amostragem probabilística: um curso introdutório. São Paulo: EDUSP; 2001.

20. Capurro H, Konichezky S, Fonseca D, CaldeyroBarcia R. A simplified method for diagnosis of gestational age in the newborn infant. J Pediatr 1978; 93:120-122.

21. Sim J, Wright CC. The kappa statistic in reliability studies: use, interpretation, and sample size require ments. Phys Ther 2005; 85:257-268.

22. Jekel JF, Elmore JG, Katz DL. Epidemiologia, bioestatística e medicina preventiva. Porto Alegre: Artmed; 2005.

23. Northam S, Polancich S, Restrepo E. Birth certificate methods in five hospitals. Public $\mathrm{H}$ ealth Nurs 2003; 20:318-327.

24. Adams M. Validity of birth certificate data for the outcome of the previous pregnancy, Georgia, 19801995. Am J Epidemiol 2001; 154:883-888.

25. M ascarenhas MDM, Rodrigues MTP, Monte NF. Caracterização dos partos e dos nascidos vivos em Piripiri, Piauí, Brasil. Rev Bras Saúde M atern Infant 2006; 6:175-181.

26. Haidar FH, Oliveira UF, Nascimento LFC. Escolaridade materna: correlação com os indicadores obstétricos. Cad Saude Publica 2001; 17:1025-1029.

27. Gould JB, Chavez G, Marks AR, Liu H. Incomplete birth certificates: a risk marker for infant mortality. Am J Public Health 2002; 92:79-81.

28. Watkins M L, Edwards L, M cClearn A, Mullins L, Mulinare J, Khaury $M$. The surveillance of birth defects: the usefulness of the revised US standard birth certificate. Am J Public Health 1996; 86:731734.

Artigo apresentado em 11/04/2008

Aprovado em 19/08/2008

Versão final apresentada em 29/10/2008 